\title{
Tu-P09-04
}

\section{Optimizing Acquisition Parameters for MASW in Shallow Water}

\author{
G. Diaferia* (Deltares), P.P. Kruiver (Deltares) \& G.G. Drijkoningen (Delft \\ University of Technology)
}

\section{SUMMARY}

Analogous to the use of Rayleigh waves in MASW on land, Scholte waves can be used to derive shear wave velocity profiles for the subsurface under water. These profiles are useful for dredging operations, offshore wind farms, oil rigs and pipelines.

We have determined the optimal acquisition set up for the detection Scholte waves in shallow water. The nearest offset, receiver spacing and number of channels have been varied in a finite difference model consisting of a water layer on top of a sediment layer and a half space. The best configuration is a nearest offset of $10 \mathrm{~m}$, receiver spacing of $1 \mathrm{~m}$ and 48 channels.

When the seafloor is not horizontal, the source/receiver spread should follow the topography. When the distance between the source and the sea floor becomes too large, no Scholte waves are detected and subsequently no reliable Vs profile can be retrieved.

In order to test the acquisition set up for a more realistic subsurface, a dipping layer and a filled channel structure were modeled. Whereas the inversion results for the dipping layer were satisfactory, the channel structure displayed large differences relative to the model, especially on the flanks. 


\section{Introduction}

On land, the determination of shear wave velocity $\left(\mathrm{V}_{\mathrm{s}}\right)$ profiles by acquisition of source-generated, dispersive surface waves (MASW) is a well-developed and established technique for geotechnical and engineering purposes (Park et al., 1999). The shear wave velocity is directly correlated to the stiffness of a soil. In shallow marine environments, information regarding the stiffness of the shallower part of subsurface is equally relevant. The knowledge of the variation of shear strength with depth can be used when planning the locations of offshore wind-farms, oil-rigs or pipelines.

Surface waves in the underwater environment are called Scholte waves (Scholte, 1947). These waves travel along the sea-floor with slightly lower velocities than those of Rayleigh waves on land due to the influence of the water layer. For Scholte waves, the fundamental mode of the dispersive surface waves can be picked and inverted obtaining a $\mathrm{V}_{\mathrm{s}}$ profile of the subsurface.

Scholte waves cannot always be successfully recorded, unless a proper acquisition setup is chosen. The aim of this research is to retrieve the best combination of acquisition parameters to be able to detect Scholte waves in shallow marine environments. Acquisition parameters like nearest offset, receiver spacing, spread length and depth of the streamer were investigated using a synthetic model.

\section{Methodology}

In order to test the best acquisition parameters for Scholte waves in a shallow marine environment, a simple, laterally homogeneous model is implemented where a low-velocity $\left(V_{\mathrm{s}}=200 \mathrm{~m} / \mathrm{s}\right) 4 \mathrm{~m}$ thick layer overlays a higher velocity $\left(\mathrm{V}_{\mathrm{s}}=600 \mathrm{~m} / \mathrm{s}\right)$ half-space, with a sea floor at $6 \mathrm{~m}$ depth. Additionally, the velocities are decreased (to $100 \mathrm{~m} / \mathrm{s}$ and $300 \mathrm{~m} / \mathrm{s}$, respectively) to study the effect of low velocities of the subsurface on the acquisition. For each model, synthetic data have been generated with a finitedifference method in order to solve the 2D wave equation. After filtering, the dispersion curves were calculated in the velocity-frequency domain. Subsequently, the Geopsy ${ }^{\circledR}$ package was used for inversion to $\mathrm{V}_{\mathrm{s}}$ profiles.

In the first part of the study, source and receivers were kept at a constant distance of $3 \mathrm{~m}$ from the sea floor, sufficient to generate and acquire Scholte waves as shown in Kruiver et al. (2010) and El Allouche et al. (2011). The acquisition parameters allowed to vary were nearest offset $\left(\mathrm{x}_{1}\right)$, receiver spacing $(\Delta x)$, spread length $(x)$ and variation of depth of streamer, related to depth of sea floor. In the second part of the study, lateral heterogeneities of the subsurface were introduced.

\section{Results}

\section{1 - Variation of nearest offset}

The position of source relatively to the first receiver can have a strong influence on the recorded surface waves. Generally, near-offset effects are observed when the source is not sufficiently far from the receiver spread leading to an incoherence of surface waves events in a shot gather. To avoid this effect in the MASW case (on land), Stokoe et al., (1994) propose a nearest offset of at least half of the minimum wavelength. To study the effects of nearest- and far-offset, 20 shots were modelled with a nearest offset ranging from $0 \mathrm{~m}$ to $80 \mathrm{~m}$.

According to the rule of thumb mentioned above, near-offset effects should not be observed for our model for $\mathrm{x}_{1} \geq 27 \mathrm{~m}$. The dispersion plots are shown in Fig. 1 (left). In the near-offset case ( $\mathrm{x}_{1}$ $=0 \mathrm{~m}$ ), the fundamental mode is less resolved compared to the larger offset cases. From the dispersion curves, it appears that a nearest offset $\mathrm{x}_{1}=5 \mathrm{~m}$ is sufficient to avoid an undesired nearoffset effect. According to the inversion results (Fig. 1, right), a good compromise is a nearest offset value of $10 \mathrm{~m}$ since both depth and shear wave velocities of the two layers are well 

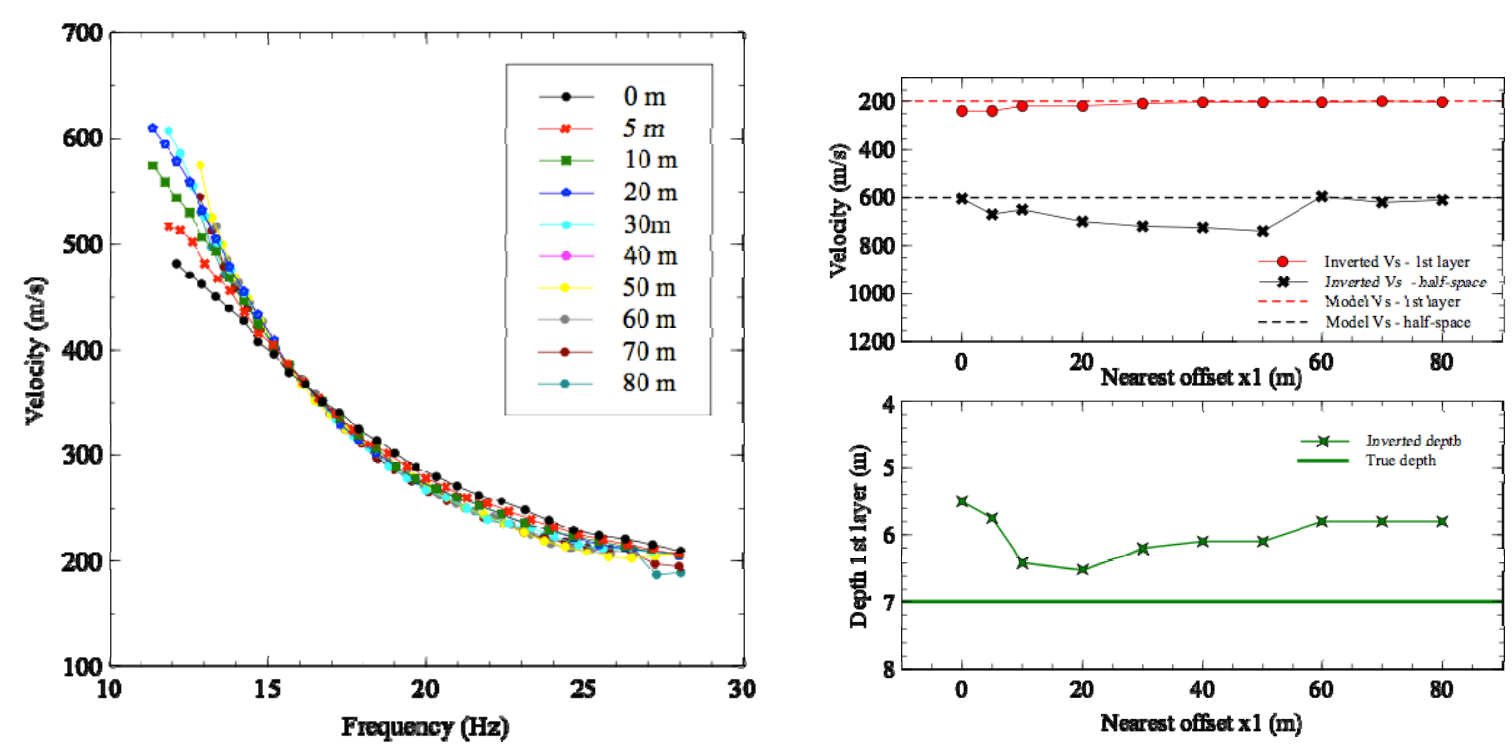

Figure 1 Left: Picked fundamental mode for nearest offset $\left(x_{1}\right)$ ranging from 0-80 m. Right: inversion results (Shear waves velocities, depth of the interface between layer 1 and half-space) depending on nearest offset $(0-80 \mathrm{~m})$.

constrained. Moreover, both fundamental mode and $1^{\text {st }}$ higher mode are sufficiently sharp resolved in the dispersion (v,f) plot to allow simple and less ambiguous picking.

\section{2 - Variation of receiver spacing}

The distance between receivers controls the spatial resolution of the sampled wave field. As a rule, spatial sampling has to be at least half of the minimum wavelength (in our case around $3 \mathrm{~m}$ ) in order to avoid spatial aliasing.

According to the results obtained in the previous section, a value of $\mathrm{x}_{1}=10 \mathrm{~m}$ for nearest offset was used. Four different values of receiver spacing were tested $(\Delta x=0.5 \mathrm{~m}, 1 \mathrm{~m}, 2 \mathrm{~m}$ and $4 \mathrm{~m})$. In the $(\mathrm{v}, \mathrm{f})$ spectrum the fundamental mode was hardly affected by a spatial-aliasing artefact. The automatic picking resulted in identical dispersion curves and identical inversion results for all cases. In conclusion, for the standard model, a receiver spacing up to $4 \mathrm{~m}$ is sufficient to correctly retrieve the fundamental mode.

The required receiver spacing to avoid spatial aliasing strongly depends on the $V_{s}$ of the subsurface for the same input frequency: the lower $\mathrm{V}_{\mathrm{s}}$, the smaller the wavelength and the smaller the required spacing. The modelling results with a $50 \%$ decreased $\mathrm{V}_{\mathrm{s}}$ velocity, however, still show a good resolution even with the coarsest receiver spacing. This is because of the low frequency content of the modelled Scholte waves.

\section{3 - Variation of spread length}

Four different spread lengths (with 24, 48, 72 and 96 channels, respectively) have been tested for a fixed nearest offset $\left(\mathrm{x}_{1}=10 \mathrm{~m}\right)$ and a receiver spacing $(\Delta \mathrm{x}=1 \mathrm{~m})$. Better results are obtained for longer receivers spreads, because different phase velocities have the possibility to better separate as the wave field propagates in space. For longer spread lengths, the fundamental mode and higher modes have a sharper defined energy in the (v,f) spectrum (Fig. 2, left). The inversion results (Fig. 2, right) show that 24 channels are not sufficient to retrieve a reliable $\mathrm{V}_{\mathrm{s}}$ profile of the subsurface. A good compromise is achieved already with 48 channels.

Summing up, it is demonstrated that the best compromise for Scholte wave acquisition consists in an acquisition set up with 48 channels at $1 \mathrm{~m}$ spacing with a nearest offset of $10 \mathrm{~m}$. Modelling 
results show that even in the case of encountered slower shear-wave velocities, the aforementioned configuration appears to be suitable.

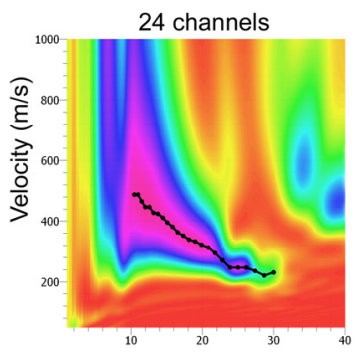

72 channels

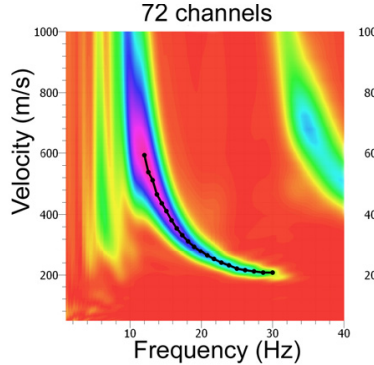

48 channels

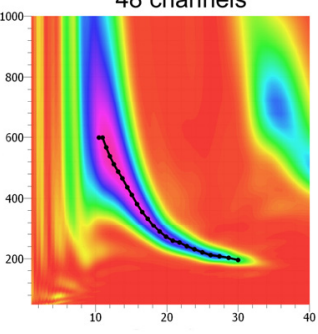

96 channels

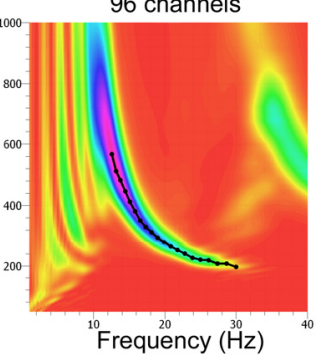

Figure 2 Left: Phase velocity plots for different spread lengths for the standard model with $x_{1}=10 \mathrm{~m}$ and $\Delta x=1 \mathrm{~m}$. Right: inversion results depending on number of channels (24 to 96). Top: shear wave velocities, bottom: depth of the interface between layer 1 and half-space.

\section{4 - Variation of depth of sea floor}

A key issue in Scholte way acquisition is presented by the influence of the water layer between source/receivers and the sea floor over the generation and recording of surface waves. The source has to be close to the water bottom in order to generate shear waves and Scholte waves. To investigate the influence of the distance to the sea floor, two different configurations were tested for a gently dipping $\left(4.6^{\circ}\right)$ sea floor:

- source and receivers always at the constant depth

- source and receivers "following" the sea-floor topography

The inversion results of the first configuration demonstrate that as soon as the distance of the source from the sea floor becomes larger than $9 \mathrm{~m}$, the quality of the dispersion curves strongly decreases, making it impossible to retrieve correct values for $\mathrm{V}_{\mathrm{s}}$ and depth. For the second case, where the source is always at $3 \mathrm{~m}$ above the sea floor, the $V_{\mathrm{s}}$ and depths were recovered reliably. Concluding, the source height needs to be adjusted when dealing with a non-horizontal sea floor in order to have a suitable generation and acquisition of Scholte waves.

\section{5 - Variation in the subsurface}

In all the above examples, the dispersion curves are inverted to a $\mathrm{V}_{\mathrm{s}}$ profile assuming variations in wave speed in the vertical direction only (1D). In order to study how reliable a laterally heterogeneous subsurface can be retrieved, the starting model has been slightly modified. The influence of a gently dipping $\left(4.6^{\circ}\right)$ interface between the first layer and half-space and a sediment filled channel structure were evaluated (Fig. 3). The influence of the lateral heterogeneity is rather strong and increases with the thickening of the first layer: the velocity of the half-space is increasingly stronger underestimated (Fig. 3a). For a channel structure, the errors in recovered velocity and depth are most prominent on the flanks. It is thus shown that a 1D approach to the inversion of Scholte waves is rather prone to error when dealing with lateral heterogeneities on the scale of the spread. 

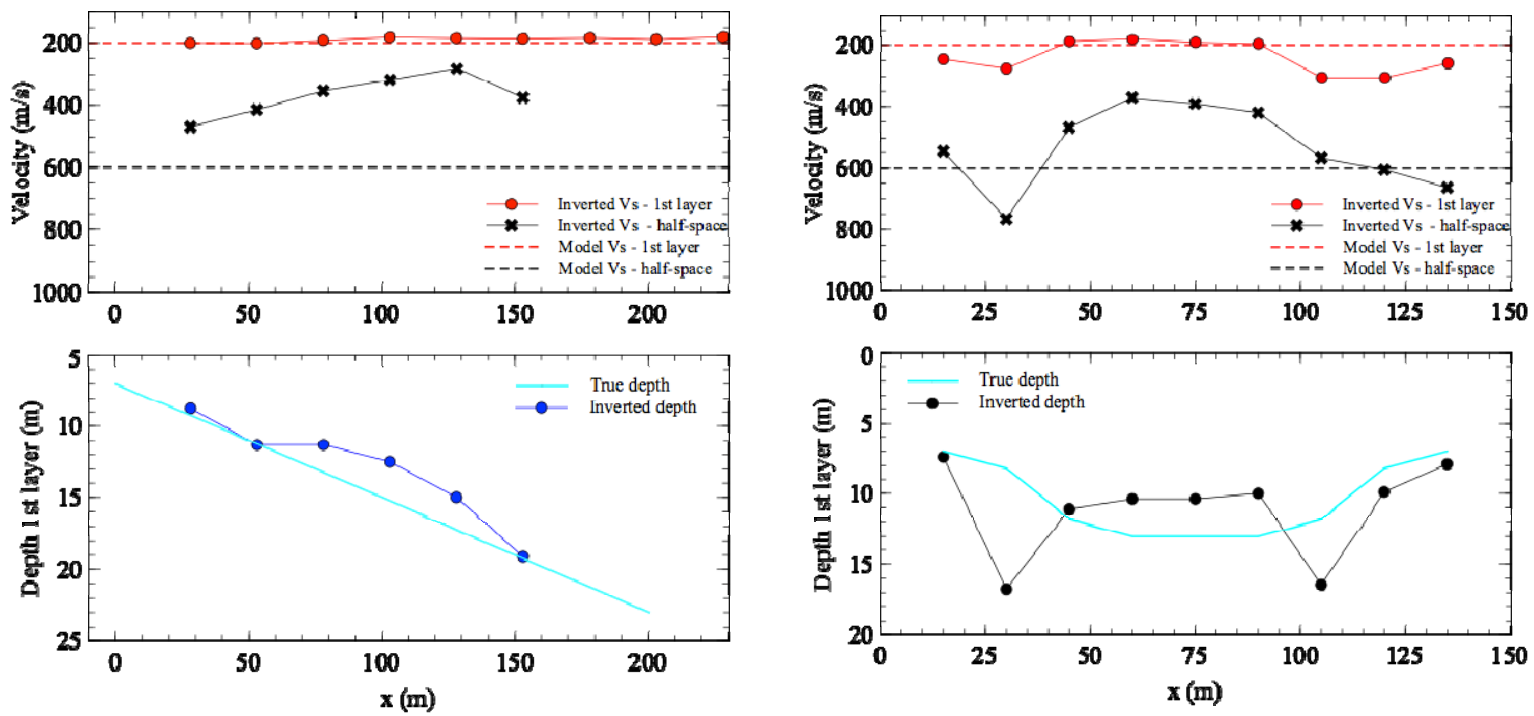

Figure 3 Left: Inversion results for a dipping interface between 1st layer and half-space. Right: inversion result for a channel structure.

\section{Conclusions}

In order to retrieve the optimal acquisition parameters for Scholte waves, a finite-difference scheme was used to model a propagating wave field in a shallow marine environment with several different acquisition set ups. The results were subsequently analysed, dispersion curves picked from the $(\mathrm{v}, \mathrm{f})$ spectrum and the dispersion curves inverted using a 1-D inversion scheme.

From the different analyses, the following optimal values were determined: nearest offset $\mathrm{x}_{1}=10 \mathrm{~m}$, receiver spacing $\Delta \mathrm{x}=1$ or $2 \mathrm{~m}$ and 48 channels. This configuration assures a good resolution of the fundamental mode in the $(\mathrm{v}, \mathrm{f})$ spectrum and minimal uncertainty in picking. In addition, it is also suitable for lower velocities in the subsurface. In case of variable sea-floor topography, the source has to follow this topography of the sea bottom in order to assure the generation and subsequent acquisition of Scholte waves.

If lateral heterogeneities are known to be present, the use of a $1 \mathrm{D}$ inversion approach can result in $\mathrm{V}_{\mathrm{s}}$ profiles that contain large deviations relative to the true values.

\section{References}

El Allouche, N., Drijkoningen, G. G., Versteeg, W. and Ranajit, G. [2011] Converted waves in a shallow marine environment: experimental and modeling studies. Geophysics, 76, T1-T11.

Kruiver, P.P., Deák, A. and El Allouche, N. [2010] Extraction of geotechnical properties from Scholte waves in underwater environments. First International Conference on Frontiers in Shallow Subsurface Technology, Extended abstract.

Park, C.B., Miller, R.D. and Xia, J. [1999] Multichannel analysis of surface waves (MASW). Geophysics, 64, 800-808.

Scholte, J.G. [1947] The range of existence of Rayleigh and Stoneley waves. Geophysical Journal International, 5, 120-126.

Stokoe II, K.H., Wright, G.W., James, A.B. and Jose, M.R. [1994] Characterization of geotechnical sites by SASW method. In: R.D. Woods, (Ed.) Geophysical characterization of sites, ISSMFE Technical Committee \#10. Oxford Publishers, New Delhi. 